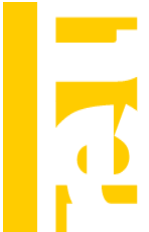

\title{
LAS REFORMAS LABORALES EN LA OBRA DE LUIS TOHARIA
}

\author{
Inmaculada Cebrián*, Gloria Moreno*, José Ignacio Pérez Infante ${ }^{\dagger}$ \\ * Universidad de Alcalá \\ $\dagger$ Economistas Frente a la Crisis
}

\section{Resumen}

Este artículo revisa las aportaciones de Luis Toharia al estudio de las reformas laborales, tanto desde el punto de vista teórico, como desde la perspectiva de los cambios más importantes que se han introducido en España desde la aprobación del Estatuto de los Trabajadores en 1980. Desde el punto de vista teórico, se hace hincapié en la insistencia de Luis Toharia sobre la dificultad de que por sí solas las reformas laborales creen empleo y sobre la imprevisión de sus resultados. Y desde el punto de vista de los cambios más relevantes se analizan los artículos de Luis Toharia sobre la reducción de la temporalidad, las medidas dirigidas a facilitar y abaratar el despido y las modificaciones del sistema de prestaciones por desempleo.

Palabras clave: Reformas laborales, temporalidad, despido, prestaciones por desempleo.

Clasificación JEL: J08, J41, J65.

\section{Abstract \\ This article reviews Luis Toharia contributions to the study of labor reforms, both from the theoretical point of view, and from the perspective of the most important changes introduced in Spain since the approval of Workers Law (Estatuto de los Trabajadores) in 1980. From the theoretical point of view, it is emphasized his insistence on how difficult is for labor reforms to create jobs and how much unpredictable are their results. It is also review some of his most interesting articles about relevant legislative changes related with reduction of temporality, measures to facilitate and reduce severance payments and modifications to unemployment insurance system. \\ Key words: Labour reforms, temporary contracts, dismissal, unemployment benefits. \\ JEL Classification: J08, J41, J65. \\ 1. El análisis teórico de Luis Toharia del mercado de trabajo y sus implicaciones en el estudio de las reformas laborales}

A lo largo de la obra de Luis Toharia se encuentran numerosas referencias a la relevancia que tiene la regulación jurídica del mercado de trabajo como un aspecto muy importante para entender el modelo social y

(c) Revista de Economía Laboral 
económico imperante en la sociedad española, lo que le convierte en un pionero en la defensa de la relación entre economía y derecho laboral en el análisis del mercado de trabajo, relación que todavía es difícil de encontrar en el estado actual de ese análisis. Pero no solo tiene importancia en la obra de Luis Toharia las referencias a la normativa laboral, sino también, y de forma muy reiterativa, el análisis de las transformaciones de esa normativa, es decir, de las reformas laborales o del mercado de trabajo.

Así, en el libro colectivo, publicado en 1998, que coordinó y dirigió Luis Toharia, El mercado de trabajo en España, al inicio del apartado titulado Los cambios del marco jurídico del mercado de trabajo español señala que "La regulación jurídica del mercado de trabajo es uno de los aspectos de la vida social española en el que mejor pueden observarse las transformaciones que se han producido en las tres últimas décadas. Sin embargo, estos cambios de la legislación laboral no sólo son interesantes per se; también nos dan una idea de la forma en que se han abordado los diferentes problemas que han aquejado a la economía española"'.

Pero, en la mayoría de las ocasiones, el planteamiento básico de las reformas laborales, la necesidad de desregular el mercado de trabajo y de flexibilizarlo en distintas líneas de actuación (flexibilidad interna y externa de las empresas, flexibilidad salarial o limitación y reducción del sistema de protección por desempleo) entraña una determinada perspectiva ideológica, es decir, una concreta forma de entender y explicar qué es el mercado de trabajo y cómo funciona. En última instancia, cuál y cómo es la mercancía que se intercambia en ese mercado, cuáles son los factores determinantes de la demanda y la oferta de esa mercancía y cuáles son los mecanismos que, principalmente, explican el nivel y la evolución del paro.

Y todos estos aspectos, cargados de ideología en la mayoría de los análisis realizados en el marco de la economía convencional u ortodoxa, fueron desmenuzados y analizados con un elegante espíritu crítico en un artículo publicado en 1987 y escrito conjuntamente por Lluís Fina y Luis Toharia en Cuadernos Económicos de ICE n $\mathrm{n}^{\circ}$ 37, titulado "La caracterización de los mercados de trabajo: relaciones teóricas e implicaciones". En este artículo, de gran relevancia para los análisis críticos de la economía laboral en nuestro país, Lluís Fina y Luis Toharia, dos grandes amigos que compartieron una misma forma de entender muchos aspectos de la vida y, en particular, la economía del trabajo y las implicaciones que la misma tiene en las políticas públicas y en las reformas del mercado de trabajo, analizan con detalle las características de la mercancía que se intercambia en el mercado de trabajo, una

${ }^{1}$ Toharia, L. (coordinador) (1998), página 144. 
mercancía muy diferente a las que se intercambian en otros mercado de bienes y servicios y que convierten al mercado de trabajo en una institución de fuertes connotaciones sociales.

Según Lluís Fina y Luis Toharia, y en la línea de algunos economistas pertenecientes a distintas escuelas o tendencias, como eran en la época que se escribió el citado artículo Solow (ortodoxo), Tarling y Wilkinson (heterodoxos), Piore (institucionalista) o Bowles (marxista), la mercancía que se intercambia en el mercado de trabajo no es el trabajo ya realizado si no la fuerza de trabajo, o "capacidad de trabajar" del trabajador. La capacidad de trabajar, que es la mercancía que se demanda y ofrece en el mercado de trabajo, debe convertirse en el trabajo realizado lo que implica que el empresario que la ha comprado utiliza normalmente todos los medios disponibles para que esa conversión sea la más beneficiosa para la empresa.

Todo ello implica que la relación laboral entre empresario y trabajador no es una mera relación mercantil sino que también es una relación social. Esta perspectiva previa del análisis del mercado de trabajo, muy diferente a la del análisis dominante en la economía convencional u ortodoxa, condiciona todo el análisis posterior y cuestiona muchas deducciones y conclusiones dominantes en la economía laboral, tanto en lo que respecta a la demanda y la oferta de trabajo como a la existencia de equilibrios o de desequilibrios en el mercado de trabajo.

Desde esta perspectiva crítica, el salario, precio de la mercancía intercambiada en el mercado de trabajo, no actúa necesariamente como un precio equilibrador entre la oferta y la demanda de trabajo, como es en el caso del resto de los mercados de bienes y servicios, sino que cumple otro papel de carácter social, por lo que en muchas ocasiones las recomendaciones basadas en una mayor flexibilidad salarial, en las que los salarios reales e incluso los monetarios deben caer en situaciones de elevado nivel de paro, chocan contra ese "papel" social de los salarios.

Y ello porque los salarios no tienen únicamente la dimensión de coste laboral, de coste de las empresas, que es la dimensión predominante y casi única de la mayoría de los análisis de la economía convencional, sino que tienen otra dimensión, al suponer los salarios la mayor parte (en muchos casos la única) de los ingresos de los trabajadores y determinar, en consecuencia, una parte sustancial del consumo privado y, por lo tanto, de la demanda global y del PIB de la economía.

Pero con la crítica de Lluís Fina y Luis Toharia a la consideración del mercado de trabajo y al análisis teórico del mismo también se deduce una crítica muy relevante a las implicaciones prácticas y políticas de la mayoría de los análisis efectuados por la economía convencional y, en concreto, por los defensores de las reformas laborales. En efecto, si el 
"problema" principal del mercado de trabajo en el análisis convencional u ortodoxo es el deficiente funcionamiento del mercado de trabajo o, lo que es lo mismo, la acumulación de distintas formas de rigideces de ese mercado, las reformas laborales deberían fundamentarse en la mejora de ese funcionamiento del mercado de trabajo y en la reducción de esas abundantes rigideces laborales, defendiendo la desregulación generalizada del mercado de trabajo y el aumento de la flexibilidad del mismo.

En este sentido, muchas de las peticiones de reforma laboral de la mayoría de los economistas y de muchos organismos nacionales e internacionales se han concretado en la defensa de reformas laborales dirigidas a esa mayor flexibilidad. Y fruto de esa insistencia ha sido la orientación de la mayoría de las reformas laborales desarrolladas a partir del Estatuto de los Trabajadores aprobado en 1980 y, en concreto, de la más amplia, ambiciosa e intensa en este sentido, que es la reforma laboral de 2012.

Esta necesidad de mayor flexibilidad laboral imperante en las reformas laborales aprobadas en España tiene distintas vertientes, como ya se ha señalado, en concreto la insistencia en la flexibilidad interna de las empresas (modificación de las condiciones de trabajo), la flexibilidad externa también de las empresas (favoreciendo los mecanismos de entrada, a través de la contratación temporal, y los mecanismos de salida, mediante la mayor facilidad y el menor coste del despido) y la flexibilidad salarial (reduciendo los salarios reales e incluso nominales ante situaciones de elevado nivel de desempleo).

Asimismo, la defensa de ese tipo de reformas laborales ha insistido en la necesidad de debilitar la negociación colectiva, al considerarse como un mecanismo perturbador del libre funcionamiento del mercado, facilitando su descentralización e inaplicación de convenios firmados anteriormente y en vigor, y en limitar las prestaciones por desempleo (endureciendo las condiciones de acceso y de mantenimiento de las mismas y reduciendo su duración y cuantía), con la pretensión tanto de incentivar la búsqueda activa de empleo por parte de las personas desempleadas como de reducir el gasto en esas prestaciones.

En este sentido de crítica a las implicaciones teóricas y prácticas de la economía ortodoxa, Luis Toharia en 1987 en el artículo "Las diferentes explicaciones del desempleo en España y sus consecuencias para las políticas de empleo"2 analiza tres de los planteamientos básicos de esa economía en concreto, el crecimiento "excesivo" de los salarios, la negativa incidencia de los "pactos sociales" y la rigidez institucional del mercado de

${ }^{2}$ Incluido en el libro publicado por la Fundación IESA "Las causas del paro en España", el famoso libro negro, fruto también de su colaboración con Lluis Fina. 
trabajo español, que explicarían, según muchos economistas convencionales, el elevado nivel de paro y la defensa de la necesidad de reformas laborales dirigidas a flexibilizar los salarios, reformar la negociación colectiva y reducir las rigideces del mercado de trabajo.

La crítica de Luis Toharia se fundamenta, en cuanto al crecimiento salarial, en la falta de evidencia empírica de que esa sea una de las causas fundamentales de la caída del empleo. En cuanto a la negativa incidencia de los "pactos sociales", es decir, la negociación centralizada (convenios sectoriales) de los salarios, que olvidaría la importancia de la situación económica de cada sector y cada empresa, la crítica de Luis Toharia se basa en que en los años anteriores a su artículo, publicado en 1987, la evolución salarial desagregada sectorialmente se adaptaba a la evolución de la productividad por ocupado (menor crecimiento salarial en los sectores en los que menos se incrementaba la productividad por ocupado y mayor aumento salarial en los sectores de mayor avance de la productividad por ocupado), en contra de lo que se podía prever del planteamiento defendido por los economistas ortodoxos. Y, por último, en cuanto a las rigideces institucionales del mercado de trabajo, sobre todo en relación con la contratación y el despido, de los que se debía derivar un elevado grado de fijeza de los trabajadores y, por lo tanto, de la antigüedad de los mismos, concluía Luis Toharia que tampoco existía evidencia suficiente, puesto que las cifras españolas de antigüedad de los trabajadores en las empresas se situaban en el momento de la publicación de su artículo en niveles relativamente comparables con los de otros países europeos.

Por otra parte, los potenciales efectos positivos sobre la creación de empleo y la reducción del paro de las reformas laborales, que se derivaría de la mayor flexibilidad del mercado de trabajo defendida por los economistas convencionales y muchos organismos nacionales e internacionales, también los cuestionaba Luis Toharia en algunos de sus últimos artículos.

En efecto, en el artículo publicado con Miguel Ángel Malo en el Libro de Gómez, V. (editor) (2009), “¿Qué se puede esperar de las reformas del mercado de trabajo?" y en el que publicó en 2011 en la revista Gaceta Sindical de Comisiones Obreras "El debate sobre las reformas necesarias para la economía española: el mercado de trabajo" 3 aparece de manera reiterada la idea de que el mercado de trabajo ni crea ni destruye empleo, sino que lo que hace es transmitir al empleo la evolución de los mercados de productos. Y ello porque la demanda de trabajo es una demanda derivada de las demandas de los productos de la economía.

3 Toharia, L. y Malo, M. A. (2009) y Toharia, L. (2011), respectivamente. 
Por lo tanto, las reformas del mercado de trabajo por sí solas no generarían empleos suficientes para resolver el problema del paro de la economía española, si no se produjera previamente una mejora sustancial de la actividad económica, que incrementara suficientemente el PIB real o en volumen de la economía. Incluso, pudiera suceder que aun con una mejora sustancial de la actividad económica no se modificara la capacidad de creación de empleo de la economía española si, paralelamente, no se produjera un cambio del modelo productivo, del modelo de crecimiento económico.

Luis Toharia insistía en que si tan importantes y limitativas eran las rigideces laborales y tan necesarias eran su superación a través de nuevas reformas laborales difícilmente podría explicarse la intensa creación de empleo, la mayor de la UE, que tuvo lugar en España en el período 1995-2007. No le cabía duda de que el mercado de trabajo no constituyó en ese período ninguna traba a la creación de empleo; se podría afirmar que la economía española funcionó mejor o peor (lo que al fin y al cabo, decía, era un juicio de valor), pero el hecho indiscutible era la importantísima creación de empleo. Y, por otra parte, el fortísimo descenso del empleo que se produjo en España a partir de 2008 quizá no tenga que ver (por lo menos, no predominantemente) con el (mal) funcionamiento del mercado de trabajo sino con otros factores, principalmente con el freno y el retroceso de la actividad económica como consecuencia de la crisis no causada por factores relacionados con el mercado de trabajo.

Luis Toharia siempre insistía, como se ha señalado, en que lo que hace el mercado de trabajo es transmitir al empleo la evolución de los mercados de producción. Este "mecanismo de transmisión" puede funcionar mejor o peor, pero para saberlo no basta con analizar los resultados del empleo y el paro sino que hay que estudiar de dónde vienen las perturbaciones que sufre la economía y cómo el mercado de trabajo transmite esas perturbaciones.

Para él, la realidad del citado "mecanismo de transmisión" obliga a considerar los factores que, desde el lado de la demanda de trabajo, están detrás de la creación o destrucción del empleo. Las medidas dirigidas a incrementar el empleo tienen que dirigirse a impulsar la demanda de bienes y servicios y/o a fomentar la oferta de esos bienes y servicios (aumentando la capacidad productiva o mejorando la competitividad de las empresas). De esta forma, cualquier cambio (como las reformas laborales), que afecten única y exclusivamente al mercado de trabajo, estará abocado al fracaso si no va acompañado de medidas que incentiven la creación de empleo, desde el lado de la demanda y/u oferta de bienes y servicios, y, por lo tanto, se fomente la producción de la economía. 
No hay recetas mágicas ni soluciones fáciles e inmediatas decía Luis Toharia. Pero, claro, insistía que tampoco no hacer nada es garantía para frenar la destrucción de empleo ni para facilitar la recuperación cuando ésta se produzca. Por todo lo señalado, cualquier reforma laboral debiera prever los eventuales efectos negativos que sobre diferentes colectivos puede tener y ofrecer soluciones para los problemas previsibles que traerá consigo cualquier cambio normativo.

Es más, en varios trabajos suyos, en los que colabora con Miguel Ángel Malo, como en un artículo publicado en 1999 en Cuadernos de Información Económica ${ }^{4}$ y en el libro publicado también en 1999 por el Ministerio de Trabajo y Asuntos Sociales ${ }^{5}$, criticaba dos elementos muy relevantes en los planteamientos de las reformas laborales, el de incorporar a la regulación laboral española, sin más, mecanismos del mercado de trabajo de otros países y la independencia entre las distintas regulaciones e instituciones del mercado de trabajo.

En relación con la introducción de cambios para adaptar la regulación del mercado de trabajo español a la de otros países, en los dos trabajos citados se avisaba de la dificultad de copiar miméticamente el derecho laboral ajeno sin tener en cuenta la tradición y el modelo de la normativa española en materia de relaciones laborales por la inercia que ese modelo provoca en el comportamiento de los agentes sociales, sobre todo en determinadas materias, como es la práctica empresarial de los despidos disciplinarios improcedentes predominantes en nuestro país sobre los despidos procedentes por causas económicas.

Y, por otro lado, en relación con la posible independencia entre las distintas regulaciones e instituciones del mercado de trabajo, se defendía lo contrario, la interdependencia de esas regulaciones e instituciones, y se anunciaba que las reformas laborales de determinados aspectos aislados del mercado de trabajo podían provocar efectos no previstos en el conjunto del mercado de trabajo, como pueden ser las consecuencias de la flexibilidad de la contratación temporal que, al reforzar el poder de negociación de los trabajadores contratados indefinidamente, podría favorecer la aparición de rigideces salariales, independientemente de la situación económica de las empresas y del conjunto de la economía.

En este sentido, en otro trabajo de Luis Toharia y Miguel Ángel Malo, publicado en 1997 y titulado "Economía y Derecho del Trabajo: las reformas laborales de 1994 y 1997"6, se planteaban dos problemas importantes para el análisis de las reformas laborales. El primero, la

\footnotetext{
${ }^{4}$ Malo, M. A. y Toharia, L. (1999 a)

${ }^{5}$ Malo, M. A. y Toharia, L. (1999 b)

${ }^{6}$ Malo, M.A. y Toharia, L. (1997)
} 
necesidad de superar la situación en que tanto desde el Derecho como desde la Economía se han dado mutuamente la espalda sin buscar un enfoque integrador que permita averiguar cómo debe cambiarse la normativa laboral para generar ciertos efectos en el mundo económico y qué aspectos de la esfera económica necesitan de una determinada regulación para conseguir mejores resultados.

El segundo problema planteado en ese artículo de 1997 es también de gran relevancia al prever la posibilidad de que a través de nuevas reformas laborales se pueda acabar modificando el modelo de relaciones laborales establecido por el Estatuto de los Trabajadores de 1980, al alterar las relaciones de poder entre empresarios y trabajadores, algo que ha llegado a un punto extremo con la última reforma laboral, la de 2012, ya que en el objetivo principal de la normativa laboral de conseguir un equilibrio entre la libertad de empresa y la tutela de los trabajadores, puede estar desmoronándose ese equilibrio a favor de la libertad de empresa y en detrimento de la tutela de los trabajadores.

Por otro lado, Luis Toharia plantea que, como norma general, cualquier reforma laboral debería crear menos problemas que los que soluciona y que tampoco pueden esperarse cambios cruciales y rápidos gracias a modificaciones de la regulación legal. El que la regulación del mercado de trabajo pueda alterarse con cierta velocidad frente a otros instrumentos no puede equipararse a que los efectos sean inmediatos y "grandes" ya que los distintos agentes sociales y, en concreto, las empresas y los trabajadores usan la normativa legal de forma estratégica en la persecución de sus propios intereses y que, por tanto, aprovecharán (cada uno según su capacidad de poder en el conjunto de las relaciones laborales) cualquier resquicio jurídico para conseguir los mejores beneficios y/u obviar las situaciones que puedan perjudicarles.

Y Luis Toharia termina los dos artículos publicados en 2009 y 2011, citados anteriormente, con una frase que creemos que es redonda y definitiva: "Normalmente, los únicos efectos "grandes" que suelen tener los cambios normativos del mercado de trabajo son precisamente aquellos que nadie previó, como la gran extensión de los contratos temporales a partir de la reforma de 1984 o el impacto en el nivel y la distribución de los despidos que provocó la reforma de 2002", y justamente eso, que los efectos más relevantes no sean los previstos por el Gobierno, es lo que puede suceder, según el planteamiento de Luis Toharia, con la reforma laboral de 2012, que, como ya se ha señalado, es la más amplia e intensa (diríamos que también la más radical) de todas las aprobadas desde el Estatuto de los Trabajadores de 1980.

En este sentido, en el resto del artículo se va a analizar la postura de Luis Toharia en relación con algunos de los objetivos principales de las 
reformas laborales aprobadas en España, en particular en los aspectos relacionados con:

- La reducción de la temporalidad y la dualidad del mercado de trabajo.

- La mayor facilidad y reducción del coste del despido.

- La limitación de las prestaciones por desempleo, con el fin tanto de reducir el gasto público en esas prestaciones como de incentivar la búsqueda activa de empleo por parte de las personas desempleadas.

\section{La reducción de la temporalidad y la dualidad del mercado de trabajo}

Un tema que Luis Toharia ha analizado en muchas de sus obras es el problema de la temporalidad y la segmentación del mercado laboral que esta conlleva. Desde la perspectiva de las reformas laborales Luis Toharia estudió los efectos de los repetidos intentos por reducir la tasa de temporalidad a través de medidas directas, como la regulación de los contratos temporales, o indirectas, como la bonificación al empleo indefinido. En la bibliografía final se recogen algunos de los trabajos en los que se aborda este tema.

Nada mejor para expresar la opinión de Luis Toharia sobre la temporalidad, la dualidad del mercado de trabajo y las diferentes reformas puestas en marcha para corregirlas que sus propias palabras. A continuación vamos a recoger lo que él planteó en una de sus últimas intervenciones públicas ante los compañeros y colegas en la Fundación Alternativas el 27 de mayo de 2009. Decía así:

"Cuando se piensa en reformar el mercado de trabajo, no debe olvidarse que la temporalidad ha sido uno de los objetivos de varias reformas que se han adoptado en los últimos lustros en España. Cabe mencionar al menos tres reformas directamente encaminadas a reducir la temporalidad y otras dos que incidían indirectamente en ella. En primer lugar, la reforma de 1994 (inicialmente aprobada a finales de 1993) retocó numerosos aspectos del Estatuto de los Trabajadores, pero dos de sus cambios que más llamaron la atención fueron el intento de reducir el coste del despido de los trabajadores indefinidos, mediante diversos cambios legislativos tendentes a definir de forma más clara y fácilmente interpretable, los despidos por causa económica, al tiempo que limitaba casi hasta eliminarlo- el contrato temporal de fomento del empleo diseñado en 1984 y que había supuesto en su momento la ruptura jurídica más fuerte con el ordenamiento de la temporalidad, al romper el llamado 
"principio de la causalidad", según el cual el contrato temporal debe obedecer únicamente a actividades intrínsecamente temporales.

La segunda reforma significativa fue la de 1997 la cual, tras un acuerdo entre los agentes sociales, introdujo un nuevo contrato indefinido que conllevaba un menor coste de despido y que en la mayoría de sus supuestos suponía una reducción de las cotizaciones sociales que tenían que abonar los empresarios.

La tercera reforma, aprobada en junio de 2006, de nuevo tras un acuerdo entre los agentes sociales, retocó el modelo de bonificaciones a la Seguridad Social, al convertirlas en reducciones de cuantía fija y ampliar su duración, y estableció un plan extraordinario de conversiones de contratos temporales en indefinidos.

Hubo otras dos reformas de interés, aunque no pretendían afectar a la temporalidad sino, en ambos casos, a las prestaciones por desempleo, y ambas tildadas en su momento como "decretazos". En primer lugar, el Real Decreto Ley 1/92 que reformó el acceso a las prestaciones contributivas elevando el periodo mínimo de cotización de 6 a 12 meses, lo que conllevó un aumento de la duración mínima del contrato temporal de fomento del empleo también de 6 a 12 meses. En segundo lugar, la Ley 45/2002 la cual, al reformar la regulación de los salarios de tramitación con el fin de facilitar el acceso a las prestaciones por desempleo supuso de facto una disminución de los costes de despido de los contratos indefinidos y ellos por dos vías: de un lado, se suprimieron los salarios de tramitación y, de otro, se eliminaron en su práctica totalidad los costes de transacción asociados al despido, al convertirlo en un proceso en esencia automático. Debe señalarse que la reforma de 2006, antes mencionada, probablemente acrecentó la credibilidad jurídica de este procedimiento al clarificar uno de sus puntos, relativo al coste indemnizatorio en caso de despido económico improcedente de los contratos de fomento del empleo indefinido (que es de 33 días por año trabajado con un máximo de 24 mensualidades y no de 45 días por año con un máximo de 42 mensualidades, como en el despido improcedente ordinario).

Lo relevante de todas estas reformas es que todas ellas se han inspirado en el mismo principio: debe reducirse la brecha entre el coste laboral (directo o indirecto) de realizar un contrato temporal y el de realizar uno indefinido. Así sucedió en la reforma de las prestaciones de 1992, que intentó encarecer el contrato temporal (aunque sucedió algo inesperado: las empresas empezaron a utilizar el contrato de obra o servicio como contrato temporal para cualquier situación), la reforma de 1994, que abarató el despido, la reforma de 1997 que creó un contrato indefinido más barato en cuanto a cargas sociales y con menor coste de despido, la reforma de 2002, que abarató el coste del despido, y la reforma 
de 2006 que retocó las cargas sociales de los indefinidos y por la vía de la mayor seguridad jurídica seguramente abarató el coste del despido de los indefinidos. Y lo interesante de todas estas reformas es que apenas afectaron a la evolución de la temporalidad, con la única salvedad del salto que se produjo en el caso de la reforma de 2006 que pareció agotarse muy pronto (el descenso posterior tiene que ver con la crisis actual).

La consecuencia de lo anterior es que los niveles de temporalidad en España parecen tener poco que ver con los cambios de las diferencias de costes laborales entre trabajadores indefinidos y trabajadores temporales. Lo que se ha intentado hasta ahora en esa línea no ha funcionado. Naturalmente, eso no quiere decir que una reducción de la diferencia no vaya a funcionar en el futuro. Pero argumentar que lo que ha sucedido es que los cambios no han sido suficientemente significativos para que produzcan alteraciones es igual de indemostrable. La experiencia anterior obliga a preguntarse si ése es el enfoque correcto, ya que los datos empíricos no parecen ni confirmarlo ni desmentirlo. Por supuesto, siempre queda la opción "semántica": que todos sean indefinidos o temporales, es decir, que desaparezca totalmente la diferencia, por decreto.

Debe hacerse una nota final sobre la temporalidad. El sector público se ha convertido, sobre todo en el caso de las mujeres (aunque también en el caso de los varones), en un empleador de trabajadores temporales más significativo que el sector privado, y eso incluyendo a los funcionarios entre los indefinidos. Las lógicas del sector público son diferentes, sin duda, pero la rigidez para contratar en el sector público y la carga permanente que supone la funcionarización han llevado a recurrir al contrato temporal en una parte significativa de la Administración. Pero si existen funcionarios y van a seguir existiendo, debería pensarse en la posibilidad de un régimen específico de contratación diferente del concebido para un sector privado en el que dichos funcionarios no existen, al menos no legalmente._Pero en muchas ocasiones no es la normativa laboral la que dificulta el recurso a figuras indefinidas (funcionariales o no) sino otras normas de funcionamiento $y$ financiación de las administraciones públicas.

A la argumentación anterior se le puede replicar que el hecho es que el empleo en España ha disminuido mucho más que en otros países ${ }^{7}$ y que eso se debe a la fuerte presencia de contratos temporales. Suele aludirse a la injusticia que supone esa dicotomía entre el ajuste de los trabajadores temporales y la de los que tienen contratos indefinidos, totalmente protegidos por su situación jurídica. Estos argumentos pueden

7 Este comentario hace referencia a la situación vivida desde la llegada de la crisis actual. 
ser correctos, pero no parecen tener en cuenta algunos aspectos importantes de la experiencia del mercado de trabajo español tanto desde el punto de vista de la salida del empleo como de la entrada en el empleo.

Desde el punto de vista de la salida del empleo, el ajuste del empleo, en la primera parte de la crisis, estuvo muy vinculado al sector de la construcción. No es muy de extrañar, pues, que los empleos que se perdieran, los ligados a la construcción, fueran temporales, como lo son la gran mayoría de los empleos en dicho sector. Por otra parte, en el caso de la industria, también fueron los temporales los que más disminuyeron, aunque también hubo pérdida de empleo indefinido. En el caso de los servicios, en cambio, se perdió empleo temporal pero aumentó de forma visible el empleo indefinido. El caso más significativo fue el de los servicios "tradicionales" (comercio, hostelería, transportes y comunicaciones), en el que el descenso de empleo temporal fue casi totalmente compensado por un aumento del empleo indefinido.

La evolución en el trimestre más reciente ${ }^{8}$ (entre el $4^{0}$ de 2008 y el $1^{\circ}$ de 2009), en el que hubo una fortísima destrucción de empleo fue algo diferente en el sentido de que hubo pérdidas tanto de empleos indefinidos como de empleos temporales $\mathrm{y}$, sobre todo, hubo una fuerte pérdida de empleo por cuenta propia (un sector ajeno al mercado de trabajo, pero muy significativo desde el punto de vista de la actividad empresarial).

En suma, pues, puede decirse que una gran parte de la pérdida de empleo temporal, pero no toda, estuvo asociada al sector de la construcción, en el que la actividad tiende a ser intrínsecamente temporal. Pero ¿y en el caso de las empresas de los demás sectores? ¿Por qué se perdió principalmente empleo temporal? La respuesta evidente es que el coste de ajuste de los trabajadores temporales es muy inferior al del coste de ajuste de trabajadores indefinidos. Es un argumento incontestable.

Pero hay un argumento más general que afecta principalmente a las empresas cuya actividad no es específicamente temporal. Todos los estudios sobre temporalidad y empleo indefinido han ilustrado empíricamente que si hay una variable que discrimina entre una y otra situación, ésa es la ocupación o la cualificación. Simplificando, a los trabajadores poco cualificados generalmente se les hace un contrato temporal y a los más cualificados un contrato indefinido. Los primeros son poco valiosos desde el punto de vista de la productividad y seguramente son fácilmente reemplazables por lo que la empresa no tiene ningún interés en retenerlos (y por cierto en los que no tiene interés en invertir en $\mathrm{su}$ formación). Los segundos en cambio son valiosos por ser más productivos y la empresa querrá retenerlos, y una forma de conseguirlo es

${ }^{8}$ Este texto fue escrito por Luis Toharia en el año 2009. 
ofrecerles una prima en forma de contrato indefinido. Por lo tanto, no tiene nada de sorprendente que si se produce una necesidad de ajuste de las plantillas, los contratados temporales sean los primeros en ver su empleo extinguido. Si eso no sucediera sería una prueba de que la temporalidad afecta a todos los trabajadores por igual y se usa de forma casi aleatoria.

$\mathrm{El}$ argumento anterior también es válido para la contratación. Una empresa que tenga que contratar ofrecerá contratos indefinidos en primer lugar a sus trabajadores potencialmente más valiosos y difíciles de sustituir. A los menos cualificados le puede acabar interesando retenerlos mediante contratos indefinidos si, con el tiempo, van adquiriendo una cualificación específica de su puesto que los acaba haciendo valiosos para la empresa. Es para estos trabajadores para los que puede tener sentido el argumento de que el salto del coste de la extinción aumenta bruscamente cuando pasan de temporales a indefinidos. De hecho, se dice que ese salto es lo que provoca que muchos de estos trabajadores sean mantenidos en puestos temporales y no sean convertidos en indefinidos.

Se trata de un argumento razonable y el éxito de los programas específicos de conversiones subvencionadas de trabajadores con contrato temporal en contratados indefinidos así lo sugieren. Pero no puede pensarse ni mucho menos que toda la temporalidad es de este tipo. De hecho, se puede pensar que hay, al menos, tres tipos de puestos de trabajo:

- Los que son claramente productivos y a los que las empresas ofrecen un contrato indefinido, incluso de los que tienen el coste de despido más alto legalmente posible

- Los que son potencialmente productivos y a los que las empresas no se atreven a dar un contrato indefinido, porque necesitan "probarlos" en un sentido amplio del término; aunque hay veces en las que es la propia rigidez de funcionamiento de la política de recursos humanos de las empresas (y en otros casos los comités de empresa coadyuvan a esa rigidez) la que impide la creación de puestos indefinidos, generalmente considerados "fijos" por la propia empresa, puede pensarse que éstos son los puestos en los que sería deseable un fomento de la conversión o de la contratación indefinida directa

- Los que son inherentemente improductivos o inestables, para los que la empresa nunca ofrecería un contrato indefinido; estos puestos recuerdan a lo que la teoría "clásica" del dualismo del mercado de trabajo (Doeringer-Piore) llama el "segmento secundario", esos puestos necesarios para que las empresas puedan llevar a cabo sus ajustes de plantilla con rapidez y poco coste; en el caso de España habría que añadir el elemento de estacionalidad 
inherente a la actividad del país, principalmente la relacionada con el sector turístico.

Los datos sobre contratos firmados en España permiten ilustrar estas ideas y dan pistas sobre la importancia de los diferentes tipos de puestos. En primer lugar,..., el número de contratos indefinidos firmados cada año (y la tendencia de años anteriores era similar, sólo que en años más recientes se ha acentuado) y en el total acumulado en los últimos años. Entre 2003 y 2008 se firmaron 10,5 millones de contratos indefinidos, cifra similar al stock de indefinidos existente en promedio en ese periodo. Es decir, si todos los indefinidos que fueron contratados permanecieran en su puesto de trabajo, todos los que había habrían sido desplazados, por despido o jubilación. Dado que ese desplazamiento no se ha producido, lo anterior implica que acceder al empleo con un contrato indefinido en España dista mucho de ser un acceso a un contrato "fijo" o "permanente" y no digamos "vitalicio". Dicho con otras palabras, a las empresas no parece asustarles demasiado realizar contratos indefinidos a juzgar por el elevado número que se vienen firmando en los últimos años, lo que seguramente se debe a que no parece muy difícil ni costoso romper esa relación laboral. Debe añadirse que muchos de los contratos indefinidos firmados corresponden a la variedad de mayor coste de despido y que no conlleva bonificación alguna. Tampoco parece importarles a las empresas realizar esos contratos.

Por otra parte,..., el extremo opuesto al examinado no tiene que ver con la contratación indefinida sino con la contratación temporal. Se firmaron 2,7 millones de contratos temporales cuya duración pactada de antemano no llegaba a los 7 días, y se firmaron otros dos millones más cuya duración pactada no llegaba a los 30 días. Eso significa que para las empresas que realizan esos contratos, se trata de puestos inherentemente inestables y de corta duración para los que no piensan en mantener una relación mínimamente duradera. A estos puestos habría que sumar los 6 millones de contratos de duración indeterminada, muchos de los cuales corresponderán a duraciones no conocidas pero delimitadas por la duración de la obra o el servicio al que correspondan, en muchísimos casos inferior a unos meses y, desde luego, inferior a un año. Cuando se hacen propuestas de reforma del mercado de trabajo, no debe olvidarse que existe este ingente número de contratos cuya duración (corta o claramente delimitada en el tiempo) les impide pensar ni siquiera remotamente en una relación laboral indefinida.

En suma, pues, los datos sugieren que estos tipos de puestos son bien cubiertos por el mercado de trabajo español: los indefinidos directos, en su mayor parte de trabajadores relativamente más cualificados, los indefinidos por conversión ya sea con o son bonificación y ya sea con el 
mismo coste de despido o uno menor, los temporales con vocación de permanencia en la empresa y los temporales inherentemente inestables.

Un aspecto importante de lo que ha venido sucediendo en España es el que se refiere al hecho de que la entrada de contratos indefinidos en el mercado de trabajo no se ha traducido en una disminución notable del número de empleos temporales. Recuérdese que desde 2003 hasta 2008 se firmaron 10,5 millones de contratos indefinidos y, sin embargo, el stock de indefinidos sólo aumentó en 2,3 millones (entre el cuarto trimestre de 2002 y el cuarto de 2008). Aun teniendo en cuenta que en los seis años considerados se produjo un total de 3 millones de despidos y 1,5 millones de jubilaciones (altas acumuladas de pensiones de jubilación), queda un saldo de bajas no explicadas de 3,7 millones de contratos, que corresponden a otro tipo de extinción sin alta en prestaciones por desempleo y seguramente con poco coste o ninguno para el empresario. Estos datos implican que los contratos indefinidos tienen una duración muy variable y que, contrariamente a lo que a veces se oye, los contratos indefinidos no constituyen para los trabajadores que acceden a ellos una barrera de protección contra la salida del empleo.

A este respecto, tiene importancia preguntarse si la duración de los contratos guarda alguna relación con el coste laboral indirecto (cotizaciones sociales y coste del despido) de cada contrato. En efecto, una posible explicación de la deficiente acumulación de contratos indefinidos es que, a medida que se amplía el espectro de trabajadores que acceden a ese tipo de contrato, en gran medida como consecuencia de su menor coste indirecto de contratación, los contratos indefinidos corresponden a puestos progresivamente de menor productividad. Una forma de contrastar esta idea es examinar la duración efectiva de los puestos ocupados, ya que cabe pensar que la menor productividad de los puestos conllevará una duración del puesto menor. Los resultados sugieren que, en efecto, pero sobre todo en lo que concierne a las políticas de bonificación de las cotizaciones sociales, los contratos indefinidos fomentados por estas medidas tienden a tener una menor duración, lo que sugiere que corresponden a puestos de trabajo menos valiosos y productivos para las empresas".

\section{Las reformas laborales dirigidas a facilitar $y$ abaratar el despido}

Son muy abundantes los trabajos realizados por Luis Toharia, muchos de ellos en colaboración con su discípulo y amigo Miguel Ángel Malo, en los que trata la problemática y efectos de las reformas laborales dedicadas a facilitar y abaratar el coste del despido, entre ellos el libro, ya 
citado, publicado en 1999 por el Ministerio de Trabajo y Asuntos Sociales, titulado "Costes de despido y creación de empleo"9.

Una primera cuestión tratada por Luis Toharia en estos trabajos es la revisión del estado de la teoría económica sobre los efectos del coste de despido. En esta revisión, se hace hincapié en que el coste del despido por sí mismo no es un elemento que limite de forma relevante la creación de empleo y que, por lo tanto, incida en el nivel de empleo y de desempleo, y ello porque el mayor o menor coste del despido puede afectar a la entrada en el empleo (contratación) pero también a la salida del empleo (despido) y que la mayor o menor intensidad de cada uno de estos dos fenómenos dependerá de forma determinante de la situación cíclica de la economía, de que la economía se encuentre en una fase expansiva o en una fase recesiva. Por ello, en el conjunto del ciclo económico el efecto final sobre el empleo y el paro es, a priori, indeterminado y puede llegar a ser prácticamente neutro.

Sin embargo, ese factor de estabilidad en el nivel de empleo a lo largo del ciclo que puede deducirse del coste del despido no impide sus efectos sobre los flujos de entrada en el empleo y de salida del empleo y de que estos flujos sean elevados. Es decir, el nivel de empleo puede tender a estabilizarse pero los flujos relacionados con el empleo pueden ser intensos $\mathrm{o}$, lo que es lo mismo, la rotación laboral alta ${ }^{10}$.

Ahora bien, en otro artículo de los ya citado $^{11}$, se reitera en la posibilidad de que el elevado coste del despido afecte al nivel del empleo y el paro, indirectamente a través de los potenciales efectos negativos del coste del despido sobre otros aspectos del mercado de trabajo, como el mayor poder en la negociación colectiva de los "insiders", trabajadores internos o contratados indefinidamente, al "protegerse" esos trabajadores de las situaciones económicas desfavorables, ya que actuarían como "colchón" los trabajadores contratados temporalmente, y poder pactar esos trabajadores "insiders" salarios más elevados, aun en una situación económica desfavorable. En el mismo sentido, un efecto negativo sobre el empleo se puede producir cuando el elevado coste del despido puede llegar a favorecer una mayor inversión en tecnologías ahorradoras de mano de obra y más intensas en capital, aunque avisaban Toharia y Malo que el capital también tiene sus propios costes.

Pero Luis Toharia insistió mucho en esta problemática del coste del despido y no se contentó con revisar las teorías que analizaban los efectos

\footnotetext{
${ }^{9}$ Malo, M.A. y Toharia, L. (1999 b)

10 Malo, M.A. y Toharia, L. (1999 a), páginas 452 y 453, y Malo, M.A. y Toharia, L. (1999 b), páginas 13-16.

${ }^{11}$ Malo, M.A. y Toharia, L. (1997).
} 
sobre el empleo y el paro del mayor o menor coste del despido si no que analizó con detalle, como ningún otro autor español probablemente ha hecho, las teorías explicativas o justificativas de la existencia del coste del despido y de la determinación de ese coste.

Entre las teorías justificativas de la existencia del coste del despido distingue entre las teorías económicas y las teorías jurídicas. Las económicas basarían esa justificación en la eficiencia, por la mayor estabilidad en el empleo que se puede derivar de la existencia de costes de despidos, o, en otros casos, por su eventualidad para salvar o aliviar determinadas fallos del mercado, solucionando, por ejemplo, posibles problemas de subinversión en capital humano que se generarían si las empresas pueden despedir libremente a los trabajadores sin incurrir en costes.

Por su parte las teorías jurídicas que justifican el coste del despido se basan en la necesidad de que exista una causa para extinguir la relación laboral entre la empresa y el trabajador. Ahora bien, si la causa no es imputable al trabajador (como sería el caso de un despido disciplinario procedente) si no a las necesidades de la actividad productiva (despido procedente por causas objetivas) o no existiera ningún tipo de causa (despido improcedente), en esos dos casos el despido debiera ir acompañado de una compensación (indemnización) económica al trabajador. En estos casos (derivados de las teorías jurídicas del coste del despido), la indemnización tendría un carácter eminentemente distributivo y no de mejora de la eficiencia como en el caso de las teorías económicas.

Pero sobre todo, el problema principal es resolver la cuantía óptima de las indemnizaciones por despido, teniendo en cuenta tanto la óptica de cubrir las pérdidas de ingresos del trabajador como la óptica de la eficiencia de la empresa. El problema de la cuestión de explicar la cuantía de la indemnización por despido se plantea porque con un solo instrumento, las indemnizaciones por despido, se pretenden alcanzar dos objetivos diferentes, compensar la pérdida de ingreso de los trabajadores y mejorar la eficacia de la asignación de los recursos productivos de las empresas.

Frente a esta problemática, de igualar el número de objetivos y de instrumentos, algunos economistas convencionales han propuesto soluciones como eliminar directamente las indemnizaciones por despido o, en una propuesta menos radical, establecer una indemnización por despido que resulte óptima (en el sentido de la eficiencia o asignación de recursos productivos), para resolver fallos del mercado como la rotación excesiva de trabajadores en la empresa, y resolviendo el problema de la distribución de ingresos a través de otros mecanismos de compensación de 
rentas, como las prestaciones por desempleo o un sistema de rentas mínimas.

Ante estos planteamientos, Luis Toharia introduce la consideración de que el coste del despido puede convertirse realmente en un proceso de negociación entre las empresas y los trabajadores, lo que puede provocar que, en el caso español, se superen las cuantías mínimas establecidas por el Estatuto de los Trabajadores. Y es un proceso de negociación tanto en los despidos individuales por la posibilidad de presentar una demanda de conciliación, que puede terminar tramitándose si no hay acuerdo como un asunto en el Juzgado de lo Social, como en los despidos colectivos, en los que, con base en lo establecido en la Directiva de la UE de despidos (cuya primera versión se aprobó en 1975), se impone un período de consulta previo a la resolución del despido y que, con anterioridad a la reforma laboral de 2012, la normativa española exigía la autorización administrativa.

Además, este proceso de negociación de los despidos puede, en determinados casos, incidir en la indemnización del despido porque los empresarios incorporen a la negociación los costes que pueden provocar la dilatación del proceso. El carácter incierto de la indemnización que la empresa debe pagar en caso de despido puede llegar en su opinión a resultar tan importante como el hecho de que las empresas consideren altas o bajas la cuantía de las indemnizaciones establecidas en el Estatuto de los Trabajadores.

Resulta importante destacar que considerar los despidos como negociaciones entre dos partes que serán resueltas, si no hay acuerdo, por el juez (en el despido individual) o por la Administración Laboral (en el despido colectivo) ${ }^{12}$ permite construir modelos económicos conducentes a establecer cuáles son los determinantes de las indemnizaciones pactadas y que pueden superar a las establecidas en cada caso por el Estatuto de los Trabajadores.

Este planteamiento de considerar los despidos como negociaciones entre dos partes es lo que podría explicar la incertidumbre de la solución final y el que tradicionalmente en España despidos por causas económicas se hayan tramitado como si fuesen despidos disciplinarios improcedentes con una indemnización por despido (de 45 o 33 días de salario por año de servicio, según el tipo de contrato indefinido), muy superior a la

12 Eso es lo que ocurría con anterioridad a la supresión de esa autorización administrativa por la reforma laboral de 2012. Con posterioridad a esa reforma cabe la impugnación judicial, como en el caso del despido individual, aunque con canales y procedimientos diferentes en los dos supuestos. 
indemnización por despido procedente objetiva (de 20 días de salario por año de servicio) ${ }^{13}$.

Otra cuestión planteada en estos trabajos, y en los que se obtienen conclusiones diferentes $\mathrm{y}$, sobre todo, más matizadas a los de la mayoría de los trabajos realizados por los economistas convencionales o por los estudios realizados por determinados organismos internacionales (como la OCDE o la Comisión Europea), es el análisis comparativo sobre la facilitad y los costes del despido entre España y el resto de la Unión Europea y entre la Unión Europea y otros países desarrollados, que normalmente suelen concluir con un juicio desfavorable sobre el caso español, al otorgársele una situación de protección del empleo, en particular del empleo indefinido, muy superior a la de otros países ${ }^{14}$.

Ahora bien, en su obra constata que un problema común de toda la literatura que compara las legislaciones de distintos países en materia de reglamentaciones del despido y de la influencia de esas reglamentaciones en el empleo es la dificultad para saber si se está comparando información verdaderamente homogénea, dándose el caso de que en muchas ocasiones es muy difícil saber, cuando, por ejemplo, se comparan indemnizaciones por despido, si estas indemnizaciones se refieren a las efectivamente cobradas por los trabajadores o si corresponden a las mínimas o máximas establecidas en las correspondientes normativas, si se refieren a todos los trabajadores o únicamente a grupos específicos de trabajadores (como, por ejemplo, los trabajadores de cuello blanco o de cuello azul), si se incluyen solo los despidos individuales o también los colectivos o si se consideran las cantidades fijadas por la Ley o se refieren a las establecidas en convenios colectivos.

Todos estos problemas relativos a las comparaciones internacionales de las indemnizaciones por despido (lo mismo ocurriría para las comparaciones de otras características de los despidos, como, por ejemplo, sería el caso del preaviso), pueden llegar a cuestionar las conclusiones sobre el endurecimiento o facilitad del despido en los distintos países. Por lo tanto, es muy posible que todos estos problemas relativos a las comparaciones internacionales estén detrás, según Luis Toharia y Miguel Ángel Malo, de una parte de las diferencias que presentan las investigaciones correspondientes a esas comparaciones.

Aun así, en el trabajo de 1999 (b), se realiza una detallada y diríamos exhaustiva comparación internacional dentro de la Unión Europea entre España y el resto de los países de distintos aspectos relacionados con los despidos de trabajadores, como los procedimientos

${ }^{13}$ Malo, M.A. y Toharia, L. (1997), páginas 157-163.

${ }^{14}$ Malo, M.A. y Toharia, L. (1999 b), páginas 29-51. 
(notificación previa, comunicación por despido al trabajador,...) y la cuantía de la indemnización, estableciendo varios cuadros comparativos sobre la dificultad para despedir a trabajadores, en los que se ordena a distintos países según el grado de endurecimiento de cada legislación, poniéndose este trabajo en relación con otros estudios realizados por diferentes expertos y organismos internacionales.

La conclusión que de este trabajo se obtiene es que, según las distintas ordenaciones utilizadas, España es de los países de la UE con mayores costes de despido. Pero estas ordenaciones no tienen en cuenta que gran parte de las extinciones de contratos en España se corresponden con finalizaciones de contratos temporales, con costes mulos o muy inferiores a las de las indemnizaciones por despido. Si se consideraran las indemnizaciones por finalización de los contratos temporales el coste total por trabajador despedido cuyo contrato se extingue sería muy inferior para el caso español al obtenido habitualmente en esos trabajos. Además, previsiblemente, los datos considerados de los distintos países no consideran las indemnizaciones fijadas en los convenios colectivos, lo que elevaría el coste medio de despido por trabajador afectado de algunos de los países que se comparan con España.

Estas cuestiones, que agudizan los problemas de las comparaciones internacionales, dificultan la obtención de una conclusión clara y definitiva sobre las mayores o menores facilidades de los despidos en España en relación con otros países.

Pero, en cualquier caso, en las perspectivas de Luis Toharia hay que tener en cuenta una serie de características de la problemática del despido en España que la diferencia de la de otras economías europeas. Así, se insiste en las conclusiones del trabajo de 1999 (b) ${ }^{15}$ en que:

- La normativa legal del despido en España, a pesar de las cuantías establecidas en el Estatuto de las indemnizaciones, la configura como una negociación de las mismas, tanto para los despidos individuales como para los colectivos.

- Los costes de despido en España son muy diferentes según la vía utilizada para despedir al trabajador (despido disciplinario $\mathrm{u}$ objetivo, individual o colectivo) y del contrato laboral de este, lo que ha tenido dos efectos principales: la forma prioritaria de realizar despidos económicos ha sido el despido económico individual y, dentro de este, principalmente el disciplinario y la creación de un mercado de trabajo fragmentado en función del tipo de contrato laboral.

\footnotetext{
15 Páginas 134 y 135.
} 
- La diferencia de la práctica real con la legislación, debido al uso estratégico que las empresas suelen hacer de la normativa, lleva, sobre todo hasta la reforma laboral recientemente aprobada de 2012, a que la indemnización por despido improcedente (de 45 días de salario por año de servicio en el contrato indefinido ordinario y de 33 días de salario por año de servicio en el contrato indefinido para el fomento de la contratación indefinida) ${ }^{16}$ sea la cantidad de referencia a la hora de decidir o pactar indemnizaciones antes de que el caso llegue al juez. Este hecho tiende a provocar que las indemnizaciones efectivamente pagadas doblen a las establecidas por la Ley para los despidos económicos (20 días de salario por año de servicio).

Además, se pone de manifiesto en la obra de Luis Toharia en varias ocasiones la necesidad de mejorar notablemente las estadísticas sobre despidos, cuyas estadísticas son fragmentarias y, en ocasiones, están sesgadas, algo que no ha sido resuelto hasta ahora. En este sentido, propone el establecimiento de una encuesta anual, tanto a los empresarios como a los trabajadores, que resuelva los problemas existentes y que pueda proporcionar información sobre diferentes materias sobre las causas del despido, el procedimiento del despido y los costes de despido realmente pagados tanto para los despidos individuales como para los colectivos, algo muy difícil que se produzca en la actualidad, cuando es intención del Gobierno suprimir la Encuesta de Coyuntura Laboral del Ministerio de Empleo y Seguridad Social, tan encomiada y apreciada, a la vez que utilizada en sus análisis, por Luis Toharia.

\section{El sistema de prestaciones por desempleo y su reforma}

El sistema de prestaciones por desempleo ha sido objeto de análisis en muchos de los trabajos de Luis Toharia. En su obra encontramos descripciones pormenorizadas de este sistema y de las sucesivas reformas que ha sufrido, pero también y principalmente, un afán por estudiar la relación entre prestaciones y empleo. Siempre estuvo presente en su análisis la idea de poder aportar alguna evidencia que apoyara o rechazara la crítica constante al sistema de prestaciones sobre su efecto perverso en el mercado laboral al retrasar la salida del desempleo,

${ }^{16}$ Estas eran las indemnizaciones anteriores a la reforma laboral de 2012; pues en esta reforma se suprime el contrato para el fomento de la contratación indefinida y se rebaja la cuantía de la indemnización por despido de 45 a 33 días de salario por año de servicio. 
desincentivar la búsqueda de empleo, reducir la probabilidad de encontrar un empleo y aumentar la tasa de paro, incrementando el paro de larga duración. Además, en este marco analítico nunca olvidaba señalar hasta qué punto el sistema de prestaciones español cumplía con sus funciones de eficiencia, entre las que destaca su papel como seguro, y lograba la equidad que requiere su papel distributivo. En la bibliografía pueden consultarse algunas obras de Luis Toharia dedicadas a este tema.

En muchas de estas obras aparece como punto de partida una descripción del sistema de protección por desempleo; que, al igual que en la mayoría de los países de nuestro entorno, se caracteriza por la existencia de dos niveles de actuación que, de acuerdo con la normativa internacional ${ }^{17}$, se establecen como indemnizaciones contributivas y subsidios asistenciales. El sistema vigente en España tiene su origen en 1980, en el Título II de la Ley Básica de Empleo (Ley 51/1980), modificado en 1984 por la Ley 31/1984 de Protección por Desempleo ${ }^{18}$. Su estructura básica consiste en un nivel contributivo, que ofrece prestaciones que necesariamente se generan mediante una cotización previa, y un nivel asistencial, que proporciona subsidios para cubrir las necesidades del desempleado que no tiene derecho al nivel contributivo, bien por haberlo agotado, bien por no tener derecho a él.

En España, el sistema de prestaciones por desempleo cubre a todos los trabajadores afiliados a la Seguridad Social que hayan cotizado por esta contingencia. De ahí, que existen algunas excepciones como son los trabajadores que tienen contratos de formación, los funcionarios públicos, los trabajadores del servicio doméstico ${ }^{19}$ o aquellos que no tienen experiencia laboral anterior. La gestión y control del sistema la realiza el Servicio Público de Empleo Estatal (SPEE).

Para tener derecho a recibir una prestación contributiva basta con haber cotización por al menos 1 año de los últimos 6 años anteriores al momento en el que perdió su empleo. Su duración depende del tiempo cotizado, medido en meses, de modo que se deduce dividiendo por 3 el tiempo cotizado, con un mínimo de 4 y un máximo de 24 meses. Los periodos de derecho resultantes deben ser pares y cada 6 meses adicionales de cotización generan 2 meses adicionales de prestaciones. En

17 Convenios 44 y 168 y Recomendaciones 44 y 176 de la OIT, sobre el desempleo (1934) y el fomento del empleo y la protección contra el desempleo (1988).

$18 \mathrm{El}$ sistema ha sido modificado posteriormente en diversas reformas que se han llevado a cabo en 1989, 1992,1993, 2002 y la última de 2012.

19 Recientemente se ha aprobado un Real Decreto que regula la relación laboral de carácter especial del servicio del hogar familiar. Se apunta por primera vez la posibilidad de que en el futuro las personas al servicio del hogar familiar pueden acceder a un sistema de protección por desempleo. 
relación con la cuantía de la prestación se establece que durante los primeros 6 meses corresponderá al 70 por ciento de la base reguladora, y al $60 \%$ el resto del tiempo que dure ${ }^{20}$. Existen unos topes mínimos y máximos fijados como un porcentaje del Indicador Público de Rentas de Efectos Múltiples (IPREM) en función del número de hijos a su cargo. Si no tiene hijos, se establece el mínimo en el 80 y el máximo en el 175 por ciento del IPREM incrementado en una sexta parte; si tiene hijos, el mínimo es 107 y el máximo alcanza el 200 por ciento del IPREM si tiene un hijo, o el 225 por ciento si tiene más de uno, siempre incrementado en una sexta parte ${ }^{21}$.

En el caso de las prestaciones asistenciales, pueden acceder todas aquellas personas que encontrándose en situación de desempleo, hayan agotado ya su prestación contributiva o no tengan derecho a percibirla por no haber cotizado el tiempo mínimo establecido por la Ley. En este caso, el individuo además debe carecer de rentas, y en caso de tenerlas, éstas no deben superar el 75\% del salario mínimo interprofesional (SMI), excluida la parte proporcional de las pagas extras. Su duración queda condicionada por diversos factores que van desde el agotamiento de la prestación contributiva o tener derecho a ella, a tener una edad determinada o tener responsabilidades familiares, etc. La cuantía de la prestación no guarda relación con el salario recibido con anterioridad, sino que se establece en el 80 por ciento del IPREM.

En España, el sistema de prestación por desempleo cumpliría en principio con las dos funciones que se esperan. Por un lado, al ser provisto por el Estado, se estaría garantizando su eficiencia al poder acceder al seguro todos los individuos y trasladar el riesgo al Estado. Por otro lado, estaría cumpliendo con su función de equidad al desempeñar su papel de distribuidor de rentas, pues garantiza el acceso y la cobertura para todos y ayuda a equilibrar la distribución de la renta. Ahora bien, la pregunta es si tiene sentido limitar las prestaciones por desempleo con el fin tanto de reducir el gasto público en esas prestaciones como de incentivar la búsqueda activa de empleo por parte de las personas desempleadas.

Para poder responder a esta pregunta en los trabajos citados se realiza un análisis en profundidad del sistema de protección por desempleo en España, además de desmenuzarse los posibles efectos económicos, tanto a nivel macroeconómico como microeconómico.

${ }^{20}$ Reducido al 50 por ciento por un Real decreto-ley de 2012.

$21 \mathrm{Si}$ el trabajador tenía una jornada laboral a tiempo parcial, los topes se calculan prorrateando en función del porcentaje de la jornada que se realizará con respecto a la que es habitual en la empresa. 
Desde el punto de vista del análisis macroeconómico, el modelo de equilibrio del mercado de trabajo determina que el salario real de la economía depende de la tasa de paro y de las variables institucionales que rijan en la economía, entre las que se encuentran las prestaciones por desempleo y el sistema de negociación colectiva. Se supone que el poder de negociación viene condicionado por el tipo de desempleo que exista, de modo que será menor, cuanto mayor sea el peso de los trabajadores desempleados involuntarios, es decir, aquellos que desearían trabajar al salario de mercado. Si se produce una extensión del sistema de prestaciones como consecuencia de un aumento de la tasa de sustitución, aumentará en términos relativos el desempleo voluntario y descenderá el involuntario, con lo que aumenta el poder de negociación y de ahí, que también se eleve la tasa de paro. Pero además, si aumenta la tasa de sustitución, desde el punto de vista del trabajador se reducen los costes de ser despedido, por lo que aumenta su poder de negociación y nuevamente se añade un efecto negativo a la tasa de paro. Ahora bien, el sistema de prestaciones tiene también sus ventajas macroeconómicas al ayudar a mejorar los procesos de emparejamiento entre oferta y demanda, de modo que la productividad del factor trabajo se ve incrementada y consecuentemente se produce una mejora de los niveles de demanda, lo que ayuda a reducir la tasa de paro. Por último, es de sobra conocido el papel de las prestaciones como un estabilizador automático que ayuda a mantener los niveles de consumo y la demanda interna cuando no hay ingresos procedentes del trabajo.

Desde el punto de vista de la microeconomía, la teoría de la búsqueda explica de qué manera se comporta el individuo cuando está desempleado. Básicamente se trata de un sistema que establece el proceso por el cual un trabajador decide aceptar o rechazar un puesto de trabajo, dado su nivel salarial de reserva, reconociendo para ello la existencia de una serie de costes que acompañan a su decisión. Por eso, si el sistema de prestaciones, por el mero hecho de existir, aumenta el salario de reserva, como resultado de la tasa de sustitución, y reduce los costes de estar desempleado, las posibilidades de que un empleo sea aceptado descienden y aumenta el nivel de paro. No obstante, no cabe duda de que el sistema ayuda a financiar el proceso de búsqueda, aunque obliga a poner en consonancia los costes del mismo. Así, cuanto mayor sea la duración del periodo de derecho, menos intensa será la búsqueda, siendo previsible que ésta aumente hacia el final de dicho periodo. Por otra parte, el ajuste del trabajador con su empleo se puede ver favorecido. Ahora bien, para que exista este ajuste es imprescindible que haya un puesto de trabajo vacante. Si hay pocas vacantes, por muy intensa que sea la búsqueda, la probabilidad de encontrar un empleo y, por lo tanto, la probabilidad de 
salir del desempleo será reducida, al tiempo que la duración del desempleo puede aumentar.

Otra discusión presente en la obra de Toharia es la conveniencia o no de utilizar los indicadores al uso para valorar la extensión de la protección al desempleo. Normalmente, con este fin se utilizan tres indicadores. Por un lado, está la tasa de sustitución, que pone en relación la cuantía de la prestación y el salario. Por otro lado, se encuentra la duración de la prestación, que tiene que ver con el tiempo durante el cual un individuo tiene derecho a estar percibiendo la prestación. Y por otro, se encuentra la tasa de cobertura, que mide el porcentaje de parados que reciben prestaciones. Cualquier aumento de alguno de ellos se entiende, en principio, como una extensión del sistema.

No obstante, Luis puso en tela de juicio la utilización de la tasa de cobertura como un indicador para establecer objetivos en cuanto a la protección por desempleo, ya que si el objetivo fuese aumentar esta cobertura podrían activarse los efectos negativos en los incentivos a la búsqueda de empleo. Más bien, la tasa de cobertura es un reflejo de la situación de penuria en la que pueden encontrarse los desempleados lo que, en un momento dado, podría llevar a adoptar medidas extraordinarias y siempre temporales de ayuda económica complementaria.

Para entender bien este argumento, debe quedar claro que la tasa de cobertura, es decir, el porcentaje de los parados que cobran prestaciones en un momento dado, depende de que los parados no tengan derecho a cobrarlas (porque no tienen acceso a ellas, o porque las han agotado) o de que no quieran cobrarlas aun teniendo derecho (por ejemplo, para acumular mayores periodos de cotización en posibles empleos subsiguientes que les puedan dar posteriormente un mejor y mayor acceso a las prestaciones). Por lo tanto, la tasa de cobertura es un resultado, tomando como punto de partida el marco normativo, tanto del funcionamiento de la economía (desde el punto de vista de la creación de puestos de trabajo) como del comportamiento de los propios parados. Debe tenerse cuidado, en esas circunstancias, de no tomar la tasa de cobertura como la variable objetivo de la política de protección.

Por otra parte, teniendo en cuenta el razonamiento anterior, cabría plantear que el análisis de la protección debería hacerse siempre en función del tiempo que se lleva en la situación de desempleo. El análisis de la cobertura del sistema de protección no puede limitarse al cálculo de tasas cuyo valor, si bien recoge el resultado efectivo de la protección, no depende de la actuación de los poderes públicos, ya que el sistema de protección por desempleo no se puede estar retocando continuamente. Es cierto, sin embargo, que estos indicadores permiten detectar situaciones 
en las que el marco jurídico existente no está dando los frutos deseables. Con todo, las tasas de cobertura no aportan la información necesaria para entender lo que está sucediendo y si el problema proviene del marco jurídico o de la propia situación del mercado de trabajo. De ahí que se hayan planteado otros indicadores complementarios que permitan conocer mejor esos problemas y detectar situaciones en las que sería necesario intervenir en el sistema, tales como, por ejemplo, la prolongación de la duración de las prestaciones o la ampliación de los colectivos sujetos a la prestación asistencial. Pero no debe olvidarse que estas medidas deberían ser excepcionales y temporales, dados los incentivos negativos que pueden generar los sistemas de protección por desempleo en el sentido de que desincentivan la búsqueda de empleo pero, sobre todo, incentivan la rotación de la mano de obra, algo que se ve muy claramente en el caso del sistema español.

En esta línea una pregunta constante en todos los trabajos mencionados es sí realmente los períodos tan prolongados de derecho a la prestación y la "generosidad" de la prestaciones hacen que una fracción importante de personas pasen largos períodos en el desempleo. La teoría económica convencional predice que la percepción de prestaciones por desempleo supone un desincentivo a la búsqueda de empleo. Esta es la explicación teórica que se suele utilizar para explicar los resultados empíricos de que los perceptores de prestaciones por desempleo presentan una menor probabilidad de salida del paro en comparación con los no perceptores. Por tanto, el mecanismo implícito entre las prestaciones y la salida del paro sería la búsqueda de empleo, que se supone resulta influida por la percepción o no de prestaciones.

Los resultados de estos trabajos sugieren que las prestaciones no afectan a la probabilidad relativa de que los perceptores de prestaciones reciban ofertas de empleo (puesto que sus actividades de búsqueda no se ven afectadas por la percepción, en comparación con otros parados no perceptores) pero influyen negativamente en la probabilidad relativa de que las ofertas sean aceptadas (dado que la disponibilidad de los perceptores es menor que la de los no perceptores), por lo que en principio cabría pensar que la percepción de prestaciones por desempleo reduce la probabilidad de que los parados salgan de su situación y alarga la duración de los episodios de desempleo. No obstante, en sus últimos estudios se sugiere que hay un segmento de trabajadores que entran y salen del empleo muy deprisa y que quedan al margen del sistema de protección social, otro segmento de trabajadores más estables en el empleo y otros que pasan mucho tiempo en el desempleo, lo cual estaría relacionado con el fenómeno de la recurrencia. Esto lleva a que la evaluación de la eficiencia del sistema de prestaciones por desempleo deba 
realizarse adoptando una perspectiva temporal y de historia laboral más amplia, no examinando simplemente la salida del paro de los individuos. Cuando se hace esto, resultan discutibles las conclusiones de política económica acerca de los posibles efectos beneficiosos que una reducción de las prestaciones por desempleo podría producir en el nivel de paro.

Esto no quiere decir que el sistema de protección por desempleo no deba retocarse. De hecho, en el trabajo de 2009 sobre El sistema español de protección por desempleo: eficiencia, equidad y perspectiva, se pone de manifiesto la conveniencia de introducir cambios que permitan adecuar el sistema actual hacia un sistema de flexiseguridad que combine flexibilidad para las empresas y seguridad para los trabajadores (entendida en el empleo y no en el puesto de trabajo). Entre otros se señalan aquellos cambios que afecten al gasto en las políticas del mercado de trabajo, poniendo como ejemplo los países que suelen ofrecerse como modelos Dinamarca y Holanda- al destacar por un mayor gasto en políticas pasivas y de mantenimiento de rentas, pero también en políticas activas. También se propone la conveniencia de articular ambos tipos de políticas (entendida como doble derecho y no como participación en las activas a cambio de la renta que se recibe en las pasivas), concentrando al principio del período de derecho las actividades formativas y, en general, las políticas activas. Otro elemento a destacar es el interés que tiene profundizar en la personalización de los servicios de asesoramiento y seguimiento en la búsqueda de empleo y en el incremento de su intensidad, sobre todo para grupos específicos (jóvenes, buscadores de primer empleo, parados de larga duración); así como la potenciación de los servicios públicos de empleo con más recursos materiales y humanos (no hay que olvidar que la cifra de trabajadores de los servicios públicos de empleo por parado en España es una de las más bajas de Europa); e incluso al modo de financiar el SPD (caminando hacia un sistema de "experience rating") para reducir en la medida de lo posible el incentivo a utilizarlo de forma desvirtuada (transformando las cotizaciones uniformes en un impuesto sobre los despidos -o separaciones- de modo que las empresas que utilizan el SPD más intensamente contribuyan más).

\section{Bibliografía}

Cantó, O. y Toharia, L. (2003): "Las prestaciones por desempleo en España: eficiencia y equidad", Hacienda Pública Española, Monografía 2003, 125-156. 
Cebrián, I. y Toharia, L. (2007): La temporalidad en el empleo: atrapamiento y trayectorias, Ministerio de Trabajo y Asuntos Sociales, Madrid.

Cebrián, I., Hernanz, V. y Toharia, L. (2009): La temporalidad en Andalucía: nuevos datos y análisis, Instituto de Estadística de Andalucía, Sevilla.

Cebrián, I., Moreno, G. y Toharia, L. (2011): Evaluación microeconómica de los programas de fomento del empleo indefinido a partir de datos de los servicios públicos de empleo y de la Muestra Continua de Vidas Laborales. Ministerio de Trabajo e Inmigración, Madrid.

Cebrián, I., García Serrano, C., Muro, J., Toharia, L. y Villagómez, E. (1995): "Prestaciones por desempleo, duración y recurrencia del paro", en Dolado J.J. y Jimeno, J.F. (comps.), Estudios sobre el funcionamiento del mercado de trabajo español, Colección Estudios, 13, FEDEA, Madrid: 155193.

Cebrián, I., García Serrano, C., Muro, J., Toharia, L. y Villagómez, E. (1996): Protección social y acceso al empleo, Colección Estudios, 34, Consejo Económico y Social, Madrid.

Cruz, J. y Toharia, L. (dirs.) (2005): El problema de la temporalidad-Un diagnóstico, Ministerio de Trabajo y Asuntos Sociales, Madrid.

Fina, L. y Toharia, L. (1987), "La caracterización de los mercados de trabajo: relaciones teóricas e implicaciones" en Cuadernos Económicos de ICE 37(3), 81-94.

García Mainar, I. y Toharia, L. (2000): "Prestaciones por desempleo y búsqueda de empleo", Revista de Economía Aplicada, 23, 5-33.

Malo, M.A. y Toharia, L. (1997): "Economía y derecho del trabajo: las reformas laborales de 1994 y 1997”, Cuadernos Económicos de ICE, 67, 155-174.

Malo, M. A. y Toharia, L. (1999 a): "Costes de despido y legislación laboral en Europa” Cuadernos de Información Económica, 15, 55-60. 
Malo, M.A. y Toharia, L. (1999 b): Costes de despido y creación de empleo, Colección Informes y Estudios, Ministerio de Trabajo y Asuntos Sociales, Madrid.

Malo, M.A. y Toharia, L. (2005):" El coste del despido y las reformas del Estatuto de los Trabajadores", en Ruesga, S., Valdés, R. y Zufiaur, J.M. $A$ XXV años de la promulgación del Estatuto de los Trabajadores, Ministerio de Trabajo y Asuntos Sociales, Madrid, 293-311.

Malo, M.A. y Toharia, L. (2008): "La reforma de los despidos de 2002", Revista del Ministerio de Trabajo e Inmigración. Economía y Sociología, 76, 111-128.

Segura, J., Duran, F., Toharia, L. y Bentolila, S. (1991): Análisis de la contratación temporal en España, Ministerio de Trabajo y Seguridad Social, Madrid.

Toharia, L. (1997): "El sistema español de protección por desempleo", Papeles de Economía Española, 72, 192-213.

Toharia, L. (1987) "Las diferentes explicaciones del desempleo en España y sus consecuencias para las políticas de empleo" en Toharia, L. y Fina, L. Las causas del paro en España, Fundación IESA, Madrid, 69-101

Toharia, L. (coord.) (1988): El mercado de trabajo en España, McGraw Hill, Madrid.

Toharia, L. (dir) (2008): El efecto de las bonificaciones de las cotizaciones a la Seguridad Social para el empleo en la afiliación a la seguridad Social: un intento de evaluación macroeconómica, microeconómica e institucional, Proyecto de investigación FIPROS (Dirección General de Ordenación de la Seguridad Social), ref. 2007/81.

Toharia, L. (2011): "El debate sobre las reformas necesarias para la economía española: el mercado de trabajo", Gaceta Sindical Comisiones Obreras, (nueva etapa) 17, diciembre, 201-236.

Toharia, L. y Malo, M.A. (2009): “¿Qué se puede esperar de las reformas del mercado de trabajo?" en Gómez, V. (editor) La reforma laboral en España, Fundación Ortega y Gasset, Madrid, 121-156 
Toharia, L., Pérez Infante, J.I. y Prudencio, C.A. (2006): La ocupabilidad de los parados registrados y la tasa de cobertura de las prestaciones por desempleo, Informe para el Servicio Público de Empleo Estatal.

Toharia, L., Arranz, J.M., García Serrano, C., y Hernanz, V. (2009): El sistema español de protección por desempleo: eficiencia, equidad y perspectivas, Proyecto de investigación FIPROS (Dirección General de Ordenación de la Seguridad Social), ref. 2008/26.

Toharia, L., Arranz, J.M., García Serrano, C., y Hernanz, V. (2010): El sistema español de protección por desempleo y la salida del paro, Papeles de Economía Española, 124, 230-246. 